\title{
Towards Atomic Resolution Electron Microscopy with Structured Temporal Electron Illumination of Picosecond Time Resolution
}

Christian Kisielowski ${ }^{1 *}$, Petra Specht ${ }^{2}$, Bert Freitag ${ }^{3}$, Erik R. Kieft ${ }^{3}$, Wouter Verhoeven ${ }^{4}$, Jasper F.M. van Rens ${ }^{4}$, Peter Mutsaers ${ }^{4}$, Jom Luiten ${ }^{4}$, Steve Rozeveld ${ }^{5}$, Joo Kang ${ }^{5}$, Alyssa J. McKenna ${ }^{5}$, Peter Nickias ${ }^{5}$ and David F. Yancey ${ }^{5 *}$

1. The Molecular Foundry, Lawrence Berkeley National Laboratory, One Cyclotron Rd., Berkeley, CA, USA.

2. Dept. of MSE, University of California Berkeley, Berkeley, CA, USA.

3. Thermo Fisher Scientific, GG Eindhoven, The Netherlands.

4. Dept. of Applied Physics, Eindhoven University of Technology, Eindhoven, The Netherlands.

5. The Dow Chemical Company, Midland, MI, USA.

* Corresponding authors: CFKisielowski@lbl.gov and DFYancey@dow.com

In recent years it became widely recognized that electron microscopy at atomic resolution is no longer limited by a lack of resolution but ultimately by beam-sample interactions. Those unavoidably cause structural alterations and atom loss depending on a material's resistance to the probing radiation [1]. The existing limitations give rise to a dose gap that exists between the number of electrons that are needed to image a single atom in bright field mode - for example it takes $\sim 10,000 \mathrm{e} / \AA^{2}$ to image one carbon atom with the current technology - and an established electron dose of only $\sim 20 \mathrm{e} / \AA^{2}$ that cannot be exceeded without causing damage to carbon rich soft materials. Significant technological advancements currently unfold a focus on the gun area, the sample area, and on detector systems of electron microscopes as schematized in Figure 1. Specifically, structured temporal emission of electrons from the gun area [2,3] is explored, electron scattering processes in the sample are reconsidered to exploit quantum effects [4], and dramatically improved detectors are developed and deployed [5]. We take advantage of shaping electron beams in the time domain in combination with advanced detection schemes to reduce the dose gap and move towards time resolved imaging at atomic resolution to access the dynamic behavior of radiation sensitive matter. Bright field phase contrast imaging and electron diffraction are applied because these methods allow for reducing dose rates to $1 \mathrm{e} / \AA^{2} \mathrm{~s}$ or less and a parallel detection of scattering events, which minimizes recording times (see Figure 1).

Ziegler-Natta $(\mathrm{ZN})$ catalysts are analyzed in this manner [6] because they are used to commercially manufacture various polyolefins since 1956, which represent the largest-volume commodity chemicals in the world. Occasionally, a clay sample was tested (see Figure 2). We reveal pristine properties of $\mathrm{MgCl}_{2}$ at $1.7 \AA$ resolution that were previously masked by air and beam damage. Beam-induced chlorine loss and a phase transformation can be delayed up to 100 times by manipulating relaxation channels with dose rates. A threshold value for minimal material alteration is reached with beam currents of femtoamperes and dose rates below $\sim 1$ e $\AA^{-2} \mathrm{~s}^{-1}$. In these conditions, a large electron dose can be accumulated while maintaining structural integrity [7].

\section{References :}

[1] C Kisielowski, Journal of Materials Science 51 (2016), p. 635.

[2] AJ McKenna et al., Nano Letters 17 (2017), p. 3952.

[3] W Verhoeven et al., Ultramicroscopy 188 (2018), p. 85. 
[4] P Kruit et al., Ultramicroscopy 164 (2016), p. 31.

[5] G McMullan et al., Ultramicroscopy 147 (2014), p. 156.

[6] C Kisielowski et al., Adv. Func. Mater. (2019), p. 1807818.

[7] The Molecular Foundry is supported by the U.S. Department of Energy under Contract No. DE-

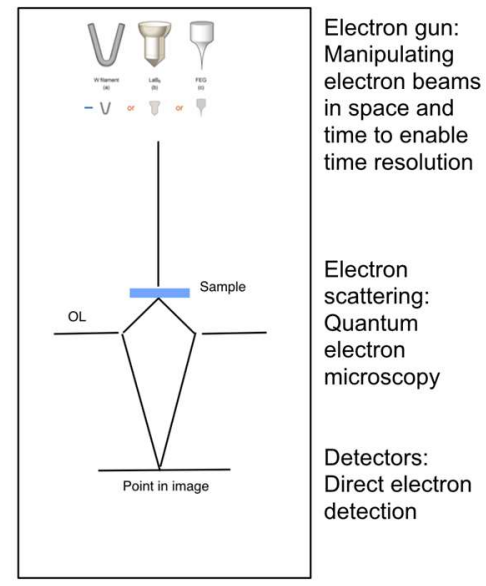

\begin{tabular}{|l|l|l|}
\hline $\begin{array}{l}\text { Relevant } \\
\text { Differences }\end{array}$ & $\begin{array}{l}\text { Broad Beam } \\
\text { preferred in } \\
\text { biology }\end{array}$ & $\begin{array}{l}\text { Focused Beam } \\
\text { Preferred in } \\
\text { material sciences }\end{array}$ \\
\hline $\begin{array}{l}\text { Acquisition } \\
\text { process }\end{array}$ & Parallel & Serial \\
\hline Dose rates & $\begin{array}{l}1-10000 \\
\text { e/Å2s }\end{array}$ & $\begin{array}{l}(6-600) \\
* 1000000 \text { e/Å2s }\end{array}$ \\
\hline $\begin{array}{l}\text { Time constants } \\
\text { (image } \\
\text { acquisition) }\end{array}$ & $0.1-10 \mathrm{~s}$ & $\begin{array}{l}1 \mu \mathrm{s}-100 \mu \mathrm{s} \\
\text { per pixel }\end{array}$ \\
\hline $\begin{array}{l}\text { Minimal spread in } \\
\text { beam direction }\end{array}$ & $<7 \AA$ & $\sim 40 \AA$ \\
\hline Detection & Real space & Reciprocal space \\
\hline Visibility & General & Selective \\
\hline Relation to theory & simple & complex \\
\hline
\end{tabular}

AC02- 05CH11231. The collaboration between Dow and LBNL was supported by a DoE CRADA.

Figure 1. Left: Areas of active research which technologically advance electron microscopy.

Right: Quantitative differences between imaging in broad beam mode (HRTEM) and focused probe mode (STEM)
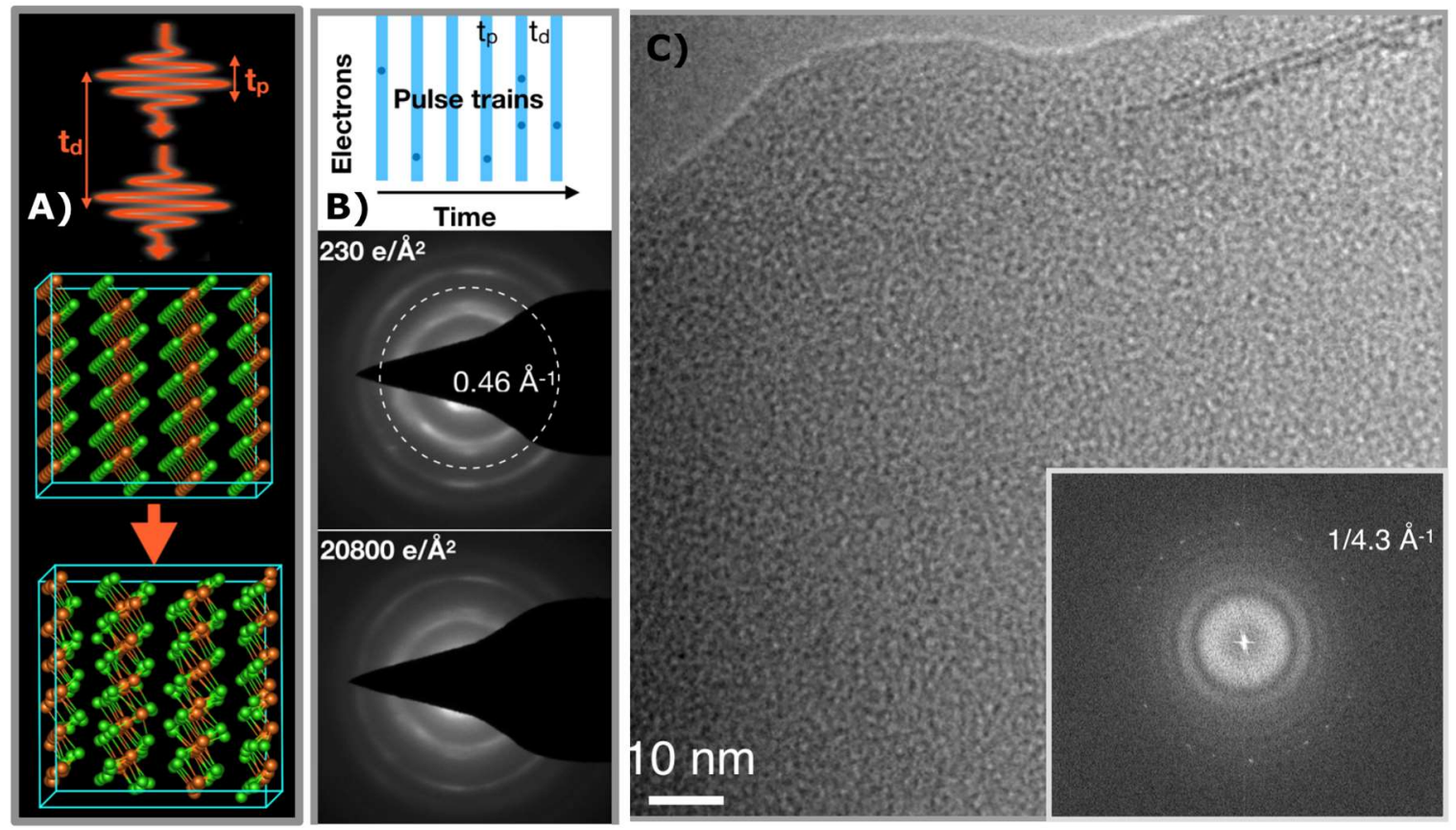

Figure 2. A) Schematics of using a pulsed electron beam for a simultaneous excitation and detection. $\mathrm{MgCl}_{2}$ is shown. Pulse length: (1.4 - 14) ps, Pulse length: (153 - 165) ps. B) Capturing the dose dependence of diffraction patterns in pulsed beam mode. C) Capturing real space images in pulsed beam mode with 1.4 ps time resolution and $4.3 \AA$ A spatial resolution (Clay sample, Courtesy: L. Whittaker, B. Gilbert, J. Banfield, Berkeley 2018) 